\title{
L'empire britannique et la conquête du Canada (1700-1713)
}

\section{Guy Frégault}

Volume 10, numéro 2, septembre 1956

URI : https://id.erudit.org/iderudit/301758ar

DOI : https://doi.org/10.7202/301758ar

Aller au sommaire du numéro

Éditeur(s)

Institut d'histoire de l'Amérique française

ISSN

0035-2357 (imprimé)

1492-1383 (numérique)

Découvrir la revue

Citer cet article

Frégault, G. (1956). L'empire britannique et la conquête du Canada (1700-1713).

Revue d'histoire de l'Amérique française, 10(2), 153-182.

https://doi.org/10.7202/301758ar d'utilisation que vous pouvez consulter en ligne.

https://apropos.erudit.org/fr/usagers/politique-dutilisation/ 


\title{
L'EMPIRE BRITANNIQUE ET LA CONQUETTE DU CANADA (1700-1713)
}

\author{
INTRODUCTION
}

Lorsque s'ouvre le XVIII ${ }^{\mathrm{e}}$ siècle, un grand fait retient l'attention: l'Amérique est en voie de prendre une place grandissante dans les affaires du monde. Dès les années 1700, les hommes d'État anglais ont le sentiment, assez confus encore, à la vérité, que les réalités américaines vont jouer un rôle prépondérant dans le nouvel équilibre de forces qu'ils s'appliquent à organiser. Comme l'a très justement remarqué un historien de l'empire britannique, si l'Angleterre prend, en 1701, la tête de la Grande Alliance contre la France, ce n'est pas surtout qu'elle craigne les suites de l'accession d'un Bourbon au trône d'Espagne; ce qu'elle ne peut pas souffrir, c'est que les Français en profitent pour s'emparer d'avantages commerciaux en Amérique du Sud et qu'ils manoeuvrent ainsi de façon à s'annexer la puissance navale de l'empire espagnol. ${ }^{1}$

Pourtant, c'est au nord du Nouveau Monde, et non pas au sud, que va se poursuivre le conflit dont l'issue décidera qui, de la France ou de l'Angleterre, se hissera au sommet de l'Occident. La raison en est simple. La colonisation ibérique est trop avancée dans le continent méridional pour que sa base essentielle, le peuplement, puisse être renversée au profit d'une autre colonisation. $\mathrm{Au}$ moment où les esprits politiques s'éveillent réellement à l'importance de l'Amérique, il est beaucoup trop tard pour que la France et l'Angleterre puissent songer, comme avait dit autrefois Montchrestien, à «planter » et à «provigner 》, au sud du golfe du Mexique, de «Nouvelles Frances» et de nouvelles Angleterres. L'Espagne et le Portugal ont pris la place.

1 Gerald S. Graham, Empire of the North Atlantic. The Maritime Struggle for North America (Toronto, 1950), 83s. 
Les sociétés auxquelles ces métropoles ont donné naissance possèdent des assises trop fermes et se sont trop développées pour que des groupes humains issus d'empires étrangers se voient jamais à même de les absorber.

Fatalement, le champ de bataille américain des deux grandes puissances européennes se dessine dans les immensités du nord. Là, tout prépare la France et l'Angleterre à se mesurer les armes à la main. Leurs ambitions les y poussent, comme aussi les craintes mutuelles qu'elles entretiennent à juste titre. La supériorité qu'elles veulent s'assurer l'une et l'autre pour dominer le monde leur commande non seulement de s'étendre le plus possible, mais aussi, et d'abord, de ruiner l'adversaire. Car, ne pas le détruire, c'est s'exposer à travailler pour lui, comme les Hollandais, avant 1664, ont travaillé au profit des Anglais. Cette conséquence découle de la manière dont l'Amérique du Nord se partage: la France y a l'espace et l'Angleterre, le nombre. A n'en juger que par les territoires qu'elle occupe ou qu'elle exploite, la Nouvelle-France semble douée d'une force incomparable. Ses frontières englobent la baie d'Hudson, l'Acadie, la vallée du Saint-Laurent (y compris les Grands Lacs) et elles viennent de se refermer sur le Mississipi, où Pierre Le Moyne d'Iberville fonde la Louisiane. L'observateur imaginaire à qui M. Toynbee fait survoler l'Amérique du Nord en 1701 peut bien conclure: «L'avenir du continent paraît virtuellement assuré; les Français seront les vainqueurs. $\gg^{2}$

Il n'est toutefois permis d'arriver à cette conclusion qu'à condition d'observer non pas la terre américaine, mais une carte bien plate et bien inerte. La vie présenterait un visage bien différent de cette œuvre de dessinateur. En réalité, dès 1700 , le peuplement de l'Amérique du Nord par les Anglais constitue un succès impressionnant. La Virginie compte plus de 58,000 habitants en 1698; le Maryland, 32,000 et le Massachusetts 60,000, en 1701. En 1680, la population du Connecticut est de 17,000 à 20,000 âmes; celle du New-York oscille autour de 18,000 en 1698, et celle du Rhode-Island atteindra 7,000 en 1708. La masse

2 Arnold J. Toynbee, A Study of History. Abridgement of Volumes I-VI par D. C. Sommervell (New-York et Londres, 1947), 97. 
anglaise s'est gonflée avec une rapidité extraordinaire. Elle comprenait 80,000 habitants en 1660 et 200,000 en 1689. En 1700 , elle est formée de 225,000 unités. ${ }^{3}$ Combien le Canada fait piètre figure auprès de ces fortes collectivités! En 1668, au moment où la France est engagée dans un effort de peuplement qu'elle ne soutiendra guère, la colonie ne renferme pas 6,000 âmes. Vingt ans plus tard, quand éclate la révolution anglaise, il n'y a pas 10,000 Canadiens. En 1698, ils demeurent en deçà de 14,000. Ils seront moins de 18,500 en $1713 .^{4}$ En 1700, le rapport entre la population canadienne et celle de l'Amérique anglaise est de 1 à 16 .

Le Canada saura-t-il jamais rattraper une telle avance ? Peut-être, à la rigueur, y parviendrait-il à peu près si la France se lançait dans une vigoureuse politique de peuplement, tout en consolidant son expansion territoriale. Mais, pour y arriver, il faudrait qu'elle suivit la voie que lui trace, au Nouveau Monde, le Canadien le plus lucide de son temps, Pierre Le Moyne d'Iberville. Elle se laisse entraîner par Iberville lorsque ce dernier préconise l'établissement de la Louisiane. Toutefois, elle fait la sourde oreille quand il demande du monde pour la nouvelle colonie. Elle ne l'écoute pas davantage lorsque, voulant profiter de l'état de guerre, le grand colonial propose d'attaquer et de détruire, à l'aide de puissantes escadres, les ports du Massachusetts, du New-York, de la Pennsylvanie, de la Virginie et de la Caroline. Iberville exprime-t-il l'idée d'une collaboration franco-espagnole en vue de fermer aux Anglais le golfe du Mexique, cette fois, c'est l'Espagne qui refuse de marcher. La stratégie du Canadien est nette: tâcher d'affaiblir l'adversaire sur la façade atlantique, donner la main aux Espagnols pour lui interdire de dominer le littoral du sud comme il domine déjà celui de l'est et, derrière lui, à l'ouest, fonder une Louisiane puissante qui, en liaison avec le Canada, le contiendrait entre les Apalaches et

3 Thomas J. Wertenbaker, The First Americans 1607-1690 (New-York, 1927), 313s.

4 Archives publiques du Canada, Archives Nationales, Colonies, série G 1 , vol. $460,4^{\mathrm{e}}$ partie: 3 (recensement de 1668) ; ibid., vol. 461: 10 (recensement de 1713); ibid., 2 (recensement de 1688). Pour le recensement de 1698, Recensements du Canada 1665-1871 (4 vol., Ottawa, 1876), 4: 39s. 
l'océan. Cependant la France se soucie peu de ces positions continentales. Elle s'intéresse davantage à ses Antilles, plus densément peuplées et d'un meilleur rapport. Tout se tient, réplique Iberville: il importe de réduire la puissance anglaise sur la terre ferme parce que "les Anglois de ce continent dans quelques années... seront en estat de transporter par le moyen de leur grand nombre de bastimens plus de vingt et trente mille hommes dans telle isle françoise qu'ils voudroient attaquer $\gg .^{5}$

Bien qu'Iberville ait alors la vue la plus nette et la plus complète du péril anglais en Amérique, d'autres personnages de la Nouvelle-France ont, à divers moments, songé à terrasser l'ennemi. En 1689, Callières avait recommandé la prise de New-York. En 1690, Frontenac avait parlé de «punir ces véritables et vieux parlementaires de Boston, de les foudroyer aussi bien que ceux de Manathe dans leur tanière $\gg .{ }^{6}$ En 1692 , le gouvernement français veut mettre à profit les connaissances que Cadillac se vante d'avoir «pour attaquer du costé de la mer la Nouvelle-York et la Nouvelle-Angleterre $\gg .^{7}$ Denis Riverin, en 1703, entrevoit le jour où les Canadiens pourront mobiliser 10,000 miliciens; alors, "vigoureux, aguerris et Entreprenans comme ils sont, Ils seront en État de renver'ser les Colonies Angloises par des Invasions Subittes et imprevües $\gg .^{8}$ A la Cour, cependant, ces idées sont loin de rallier tous les suffrages. L'ancien intendant Bochart de Champigny, maintenant l'un des principaux conseillers du ministre de la Marine, désapprouve avec conviction un raid que Philippe de Rigaud de Vaudreuil vient de faire exécuter sur la frontière de la Nouvelle-Angleterre: «Il seroit a souhaiter, opine-t-il, que cette expedition n'eut pas esté faite, $\mathrm{Mr}$ de Vaudreuil la vouloit des le temps de Mr de Calliere qui ny voulut jamais consentir, non plus que moy.» Il ajoute: «Jay une parfaite Connoiss[an]ce que les anglois ne demandent que la paix

5 Iberville le Conquérant (Montréal, 1944), 305s, 335-340, 363-389.

6 Frontenac à Seignelay, 12 novembre 1690, Rapport de l'archiviste de la province de Québec [RAPQ] pour 1927-1928 (Québec, 1928), 43.

7 Pontchartrain à Frontenac, avril 1692, ibid., 102. Voir «Mémoire du roi » à Frontenac et à Champigny, [1693], ibid., 144.

8 «Mémoire sur L'Etat des affaires de la Colonie de Canada», 18 février 1703, A.C, C 11A, 21: 278v-279. 
scachant que la guerre est Contraire a toutes les Colonies, et ce sont les françois qui l'ont toujours Commencée en Canada. » ${ }^{9}$

\section{PREMIERS PROJETS: AVANT 1708}

Est-il exact, est-il seulement vraisemblable que les Anglais «ne demandent que la paix»? En 1702, paraît à Londres le célèbre ouvrage de Cotton Mather, Magnalia Christi Americana. A un endroit, l'auteur évoque les attaques dirigées contre la Nouvelle-France par Phipps en 1690. Après avoir mentionné la conquête rapide de l'Acadie, il poursuit: «L'addition de cette colonie française aux domaines anglais n'était qu'un premier pas vers une première entreprise qui avait été l'objectif initial de Sir William Phipps et, en réalité, la plus grande tentative que les Néo-Anglais eussent jamais mise sur pied... C'était le Canada qui était la principale source des misères de la NouvelleAngleterre ... Et, de même que Caton ne pouvait pas prononcer de discours au sénat sans conclure: Delenda est Carthago, ainsi tous les esprits soucieux de la sécurité de notre pays arrivaient, en général, à cette conclusion: Il faut réduire le Canada. » Mais Québec repousse les envahisseurs. De cette campagne, Mather n'en tire pas moins un précieux enseignement: "Considérons qu'en cette affaire... il se peut que nous n'ayons vu qu'un commencement et qu'ayant appris le chemin du Canada, nous puissions édifier une victoire sur la base de ce que nous avons déjà accompli. »10 À la voix du fameux ministre, se joint, dès 1702 , celle du gouverneur du New-York, l'impopulaire lord Cornbury, ${ }^{11}$ qui, peu après son entrée en fonction, recommande à ses chefs de déloger la France de la vallée du Saint-Laurent. Six ans plus tard, il revient à la charge: "Plus je connais le pays et plus

9 Commentaire en marge d'un « Extrait de la lettre des Srs de Vaudreuil et Beauharnois du 15 novembre $1703 \gg$, ibid., 29-29v.

10 Magnalia Christi Americana: or, The Ecclesiastical History of NewEngland, from its first planting in the yoar 1620, unto the year of our Lord, 1698. First American Edition, from the London Edition of 1702 (2 vol., Hartford, 1820), 1: 167s, 172.

11 Sur Edward Hyde, lord Cornbury, voir les jugements très sévères d'Oliver Perry Chitwood, A Colonial History of America (New-York, [1948]), 301, et de Herbert L. Osgood, The American Colonies in the Eighteenth Century (4 vol., New-York, 1924), 2: 62. 
j'étudie la question, plus je vois se confirmer la facilité avec laquelle cette conquête pourrait s'effectuer. » Le Canada enlevé, raisonne-t-il, Terre-Neuve tomberait d'elle-même, et «quel avantage ne serait-ce pas pour l'Angleterre que de posséder TerreNeuve en entier »! 12

Il faut plutôt, conseille un autre Anglais, en 1703, commencer par prendre Terre-Neuve, après quoi tous les établissements français de l'Amérique du Nord tomberont d'eux-mêmes: c'est que l'occupation de Terre-Neuve fermera à la navigation française le fleuve Saint-Laurent, qui passe «derrière toutes nos colonies »; et l'occupation du Saint-Laurent «ferait autant de mal à la France que si Sa Majesté pouvait... recouvrer son ancien duché d'Aquitaine ».13 Admirable intuition! On ne saurait trouver de formule plus saisissante pour mesurer l'abaissement de la France lorsque celle-ci aura été amputée de son aile américaine. Aux yeux de ce personnage, les colonies françaises du Nouveau Monde constituent des «empiétements » sur l'empire anglais (empiétements: le mot reviendra avec une persistance monotone et sinistre dans les écrits britanniques, à la veille de la guerre de la Conquête) ; c'est ce qu'il déclare en 1704, précisant qu'il en est résulté une « grande perte nationale » pour l'Angleterre; il est urgent, souligne-t-il, de profiter des hostilités qui déchirent l'Europe pour « redresser » cette situation. ${ }^{14}$

Mais, en Angleterre comme en France, on pense d'abord aux Antilles. A la fin de 1702 et dans les premiers mois de l'année suivante, Sir Hovenden Walker - que nous retrouverons au Canada - pénètre dans la mer des Caraïbes avec une escadre destinée à emporter d'assaut la Martinique et la Guadeloupe. Son expédition avorte, bien qu'elle ait coûté fort cher et entraîné la mort d'un grand nombre de marins anglais. L'objectif, observe lord Cornbury l'année suivante, a été mal choisi. C'est le Canada

12 Cornbury au Council of Trade and Plantations, 20 août 1708, Calendar of State Papers, Colonial Series, America and West Indies, 25 (1) : 71. A l'avenir: CSPCS. - Dans les notes, nous ne transposons pas les dates des documents du calendrier julien au calendrier grégorien.

13 John Roope au Council of Trade and Plantations, novembre 1703, CSPCS, 21: 849.

14 Id. à $i d ., 27$ octobre 1704, ibid., $22: 297$. 
qu'il aurait fallu viser. Attaquer Québec, c'eût été soulager le New-York des craintes que la Nouvelle-France y inspire. Une telle campagne, à son dire, se fût révélée en définitive moins coûteuse que celle de la Guadeloupe, «outre qu'à mon avis elle eût été d'un bien plus grand avantage à la couronne d'Angleterre que n'aurait pu l'être la prise de cette île. Plus j'étudie la chose, plus je la trouve faisable $\gg .{ }^{15}$

À ce moment, Vaudreuil a mis en œuvre la politique dont il ne déviera guère: laisser en paix le New-York, afin de ne pas déranger les Iroquois, dont la neutralité s'affirme préjudiciable aux intérêts anglais, mais dévaster les frontières de la NouvelleAngleterre en liaison avec les Abénakis pour compromettre ces Indiens au point de les empêcher d'être entamés par la diplomatie ennemie. ${ }^{16}$ Le Massachusetts réplique en envoyant le colonel Benjamin Church ravager des établissements acadiens. Church fait des prisonniers, se livre au pillage, mais respecte PortRoyal. ${ }^{17}$ L'assemblée de sa province le félicite. ${ }^{18}$ Ce demi-succès met le gouverneur Joseph Dudley en appétit. Il déclare au Conseil du commerce qu'avec quatre ou cinq frégates anglaises et quelques bombes il pourrait très bien, «vu les bonnes dispositions de nos gens, faire une expédition de 1,000 hommes ou plus et, par la destruction de Québec et de Port-Royal, mettre tous les approvisionnements navals de l'Amérique du Nord entre les mains de Sa Majesté et faire cesser à jamais la guerre indigène $»^{19}$

Dans les années 1705 et 1706 , des négociations se nouent entre les gouvernements de la Nouvelle-France et de la NouvelleAngleterre. Québec et Boston échangent des missions diplomatiques qui sont censées préparer une «trêve », mais qui s'emploient

15 Cornbury au Council of Trade and Plantations, 6 novembre 1704, ibid., 307s.

16 Francis H. Hammang, The Marquis de Vaudreuil: New France at the Beginning of the Eighteenth Century (Bruges et Louvain, 1938), 118s, 174.

17 The Boston News-Letter, 12 juin 1704, 26 juin 1704, 24 juillet 1704, 7 août 1704 .

18 Ibid., 21 août 1704.

19 Dudley à Popple, 27 novembre 1704, CSPCS, 22: 325. - William Popple est secrétaire du Council of Trade and Plantations. 
visiblement à l'espionnage et à la contrebande. Comme les pourparlers lui paraissent se prolonger indûment, Vaudreuil accuse Dudley de ne chercher qu'à gagner du temps et charge «plusieurs petits partis de Sauvages d'aller en guerre dans le Gouvernement de Baston », ne doutant point «que cela ne fasse un bon effet

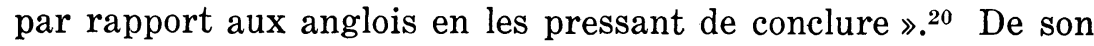
côté, Dudley proteste n'avoir pas du tout besoin d'une trêve. Il peut se défendre et même "armer assez de monde pour déloger [Vaudreuil] et tous les Français du Canada et de Port-Royal, si Sa Majesté consentait à nous fournir quatre vaisseaux de guerre et des mortiers ». Ce n'est pas tout: «Ensuite, il y aurait lieu de fonder une colonie écossaise ou anglaise à Pemaquid et à l'est. Cette partie de nos provinces est propre à nourrir 1,000 familles, et rien ne l'égale quant aux bois de construction et aux pêcheries. Un tel peuplement tuerait les espoirs qu'entretiennent les Français et les Indiens de se rétablir dans cette région au retour de la paix. ${ }^{21}$ Le Conseil du commerce goûte assez cette proposition pour la transmettre à la reine, en même temps qu'un autre plan de Cornbury. ${ }^{22}$ À la nouvelle de l'union de l'Angleterre et de l'Écosse, Dudley reprend son idée. Cette fois, il voudrait voir débarquer cinq mille Écossais en Acadie; n'y retrouveraient-ils pas le climat de leur pays natal ? Appuyés par la Nouvelle-Angleterre, ces immigrants prendraient la place des Français. Leur présence apporterait la tranquillité «sur tout le littoral américain $\gg .{ }^{23}$

Pendant que les Anglais élaborent leurs projets, les Canadiens et les indigènes frappent à coups redoublés sur les établissements ennemis. Ils n'ont pas toujours le même succès, mais ils se font craindre. Le sac de Deerfield par Hertel de Rouville, le 28 février 1704, a laissé de terribles souvenirs et des appréhen-

20 Vaudreuil et Raudot à Maurepas, 30 avril 1706, RAPQ (1938-39), 113; Vaudreuil à Maurepas, 4 novembre 1706, ibid., 168s.

21 Dudley au Council of Trade and Plantations, 1er février 1706, CSPCS, 23: 31 .

${ }_{22}$ Le Council of Trade and Plantations à Sir Charles Hedges, 4 juin 1706, ibid., 148.

23 Dudley au Council of Trade and Plantations, 2 octobre 1706, ibid., 234. Proposition bien accueillie: voir le Council of Trade and Plantations à Dudley, 26 mars 1707, ibid., 402. 
sions atroces. À la fin de janvier 1707, une dépêche de Boston laisse entrevoir des sentiments peu rassurés: «L'apparition d'un arc-en-ciel, aperçu ici samedi, le 28 décembre, vers neuf heures du matin, a fait espérer à quelques-uns que ce pourrait être un gage que Dieu ne détruirait plus Deerfield $\gg \ldots .^{24} \mathrm{Au}$ printemps, l'assemblée du Massachusetts adopte une loi portant de cinquante à cent livres la prime attachée à la capture ou à la mise à mort de tout Indien ennemi âgé de plus de douze ans. ${ }^{25}$ En maints endroits, à Piscataqua, à Marlborough, à Winter Harbour, à Exeter, à Oyster River, on signale des bandes indigènes rôdant autour d'agglomérations terrorisées. ${ }^{26}$ Le double échec du colonel John March devant Port-Royal, en juin et en août 1707, n'arrange pas les choses. La Nouvelle-Angleterre traverse une période de fatigue. Dès l'automne précédent, Dudley avait trahi de l'accablement. Tout en continuant à réclamer des forces suffisantes pour pacifier le Canada et l'Acadie, il avait demandé que, s'il n'en devait pas recevoir, il lui fût permis de conclure avec les Canadiens la trêve dont il était question depuis deux ans. Il avait expliqué: «Ce n'est pas qu'ils puissent me faire beaucoup de mal, mais ils m'occasionnent des dépenses infinies pour garder les frontières, qui retrouveraient ainsi leur sécurité; de plus, ils savent que, bien que j'aie assez de monde pour ruiner Québec, je ne peux pas y parvenir sans quatre ou cinq vaisseaux de guerre et des mortiers. ${ }^{27}$

\section{UN TOURNANT : $1708-1710$}

Les premières années de la guerre de la Succession d'Espagne sont surtout marquées par des projets de conquête venus du New-York et de la Nouvelle-Angleterre, sans que le gouvernement métropolitain paraisse se soucier d'y donner suite. À compter de 1708, cependant, la situation se modifie. La guerre tourne mal pour la France. Celle-ci cherche à négocier avec la

24 The Boston News-Letter, 20 janvier 1707.

25 Ibid., 31 mars 1707.

26 The Boston News-Letter, 23 juin, 25 août, 22 septembre, 29 septembre 1707.

27 Dudley à Sir Charles Hedges, 8 octobre 1706, CSPCS, 23: 260. 
Hollande une paix qu'elle ne réussit pas à obtenir, même au prix des conditions «les plus onéreuses ».28 Par ailleurs, des voix autorisées s'élèvent pour rappeler à l'Angleterre la valeur de son empire américain. Le Maryland et la Virginie, représente-t-on, achètent annuellement de la métropole pour 300,000 livres sterling de lainages et d'autres produits manufacturés; les mêmes colonies exportent du tabac dont la Couronne tire, tous les ans, plus de 400,000 livres sterling en revenus de douane. ${ }^{29}$ Le Massachusetts envoie en Europe, année commune, pour $\$ 30,000$ de poisson, $£ 5,000$ d'huile, $£ 2,000$ de bois de construction, $£ 10,000$ de sous-produits de la forêt, $£ 1,000$ de fourrures, sans compter le sucre, le tabac, la mélasse, le riz, les teintures que cette province reçoit des Antilles en échange de ce qu'elle y vend. ${ }^{30}$

En même temps, des esprits lucides comprennent les dangers que recèle pour le développement des colonies anglaises la politique d'encerclement esquissée par la fondation de la Louisiane. Des Bernois demandent au gouvernement anglais la permission d'aller s'établir en Amérique. Rien de mieux, répond le conseil du commerce, qui décide de les envoyer sur « la branche sud-ouest du Potomac », avec l'idée qu'une telle colonie à cet endroit « raffermira la frontière de la Virginie contre les Français du Canada et du Mississipi $\gg^{31}$ Des Palatins chassés de leur pays par les atrocités qu'y a commises l'armée française se sont réfugiés à Londres. Ils désirent, eux aussi, passer en Amérique. Le Conseil du commerce recommande de les y transporter gratuitement, de les outiller, et de les nourrir durant un an aux frais de l'Etat, après les avoir établis sur l'Hudson, où ils serviront de 《frontière contre les Français et leurs indigènes ».32 Poste dangereux: au cours de la guerre de la Conquête, les Canadiens anéantiront le village des Palatins. ${ }^{33}$

28 Louis André, Louis XIV et l'Europe (Paris, 1950), 327-329.

29 The Present State of the Tobacco Plantations, 16 décembre 1708, CSPCS, 25 (1) : 182. 235.

30 Dudley au Council of Trade and Plantations, 1er mars 1709, ibid.,

31 W. L. Grant et James Munro, éd., Acts of the Privy Council of England. Colonial Series, 2 (Hereford, 1910): 608s.

32 Ibid., $252 \mathrm{~s}, 614$.

${ }^{33}$ La Guerre de la Conquête (Montréal, 1955), 220s. 
Un officier de la Nouvelle-Angleterre fait observer en 1709 que les Canadiens s'efforcent de gagner à leur cause non seulement les Indiens de l'Acadie, mais aussi les tribus du Mississipi et toutes celles qui habitent «derrière la Caroline, la Virginie, la Pennsylvanie, le New-Jersey, le New-York, outre une partie des Iroquois ». L'Amérique anglaise est environnée de périls; «pour les écarter et pour ajouter aux avantages de l'Angleterre, il sera très nécessaire de soumettre le Canada à la Couronne ${ }^{\mathbf{3 4}}$ L'année suivante, le gouverneur Spotswood préconise de jalonner d'agglomérations le fleuve James jusqu'à ses sources. "Comme les Français, réfléchit-il, s'appliquent à établir une communication entre le Canada et leurs nouvelles positions du Mississipi par la voie des Grands Lacs, nos gens seraient en mesure, s'ils s'enfonçaient en droite ligne sur les rives du James, de couper cette communication et de s'implanter si fortement que les Français en seraient incapables de les déloger, vu surtout que l'ennemi doit faire tellement plus de chemin que nous pour atteindre ce point. » Outre que les Anglais briseraient ainsi l'armature territoriale de la Nouvelle-France, ils en profiteraient pour s'emparer de la traite de l'intérieur. ${ }^{35}$ Trois ans plus tard, un autre gouverneur, celui du New-York, attirera l'attention de ses chefs sur les efforts que fait la France pour "parsemer de colonies le Mississipi et les derrières de nos provinces », ce qui inspire la crainte qu'avec le temps «ces établissements ne nous ravissent le commerce et la soumission des indigènes $\gg .^{36}$

Ainsi, déjà se posent, et avec une remarquable netteté, les questions destinées à provoquer un conflit mondial, cinquante ans plus tard. Du simple fait de son existence, le Canada assure le maintien de l'empire français du Nouveau Monde, et celui-ci compromet le développement normal de l'Amérique anglaise. Il faut, déclare en 1708 l'agent du New-Hampshire à Londres, détruire «ces deux nids de Français», le Canada et l'Acadie,

34 « Memorial of Col. John Higginson of New England 》[30 juin 1709], CSPCS, 25 (1) : 407.

35 Spotswood au Council of Trade and Plantations, 15 décembre 1710 , CSPCS, $25(2): 316 \mathrm{~s}$.

36 Hunter à [Dartmouth ?], 14 mars 1713, ibid., 27: 157. 
c'est « le seul moyen certain de nous procurer la paix ». ${ }^{37}$ PortRoyal, reprend l'assemblée du Massachusetts, est à la NouvelleAngleterre ce que Dunkerque est à l'Angleterre; la mère-patrie devrait le conquérir ou forcer Louis XIV à le céder au prochain traité de paix. ${ }^{38}$

La déclaration la plus significative de ces années de conflit pourrait bien être, cependant, celle sur laquelle s'ouvre le fameux mémoire expédié à Londres par le colonel Vetch en 1708: ${ }^{39}$

Pour peu qu'ils connaissent la valeur du royaume britannique d'Amérique, aussi bien sous l'angle de la puissance que sous l'aspect du commerce, tous les esprits réfléchis ne peuvent que s'étonner de voir une nation aussi importante sur mer, aussi forte par le nombre et par ailleurs aussi sagement jalouse de son commerce souffrir avec autant de patience que des voisins gênants comme les Français s'installent en paix à côté d'elle, et surtout qu'avec une faible population dispersée, ils possèdent un territoire qui s'étend sur plus de 4,000 milles, encerclent et refoulent entre eux-mêmes et la mer tout l'empire britannique du continent et parviennent ainsi à réduire le commerce anglais d'un bout à l'autre de l'Amérique, en attendant de le ruiner complètement, ce qui arrivera à moins qu'ils n'en soient empêchés à temps $;.$. . ce qui rend notre attitude absolument inexcusable, c'est que la moitié de la perte qu'ils infligent en une seule année à notre économie nous suffirait, pourvu que nous dépensions cette somme à bon escient, pour les déposséder entièrement du continent et de Terre-Neuve et, par là, assurer à Sa Majesté la possession exclusive et paisible de toute l'Amérique du Nord, qui est assez vaste pour contenir quatre royaumes grands comme la GrandeBretagne.

37 George Vaughan au Council of Trade and Plantations, 6 juillet 1708, CSPCS, 25 (1) : 20.

38 Message à la reine Anne, 20 octobre 1708, ibid., 316s.

$39 \mathrm{Ibid.,}$ 41-51. Le titre de cette pièce est un véritable manifeste: «Canada Survey'd, or the French Dominions upon the Continent of America briefly considered in their situation, strength, trade and number, more particularly how vastly prejudiciall they are to the British interest, and a method proposed of easily removing them. 》 
La Nouvelle-France, poursuit Vetch est une belle proie. Ses pêcheries ont un rendement énorme. Ses fourrures sont si abondantes qu'elle en a saturé les marchés européens. Le New-York, qui exportait pour $£ 50,000$ de castor et autres pelleteries il y a vingt ans, a vu ce commerce tomber à presque rien. La crainte des sauvages soudoyés par les Canadiens réduit les colons anglais à fuir les frontières, cependant que les gouvernements provinciaux encourent de grosses dépenses pour se défendre. La Nouvelle-Angleterre est «presque ruinée ». Les alliés des Français, "que ne retiennent ni les lois de la raison ni celles de la religion », y commettent des atrocités inouïes. Les corsaires de Port-Royal désorganisent la grande pêche. Mais, ce qui inquiète Vetch plus encore, c'est que ces pertes, si graves soient-elles, ne pourraient bien être que la préfiguration d'une perte totale. En conclusion, il reprend son thème principal:

... Par les positions qu'ils ont prises, les Français ont cerné et resserré entre eux-mêmes et la mer tous les gouvernements anglais du continent, si bien qu'avec le temps, lorsqu'ils auront complètement peuplé leur pays, comme ils projettent de le faire après la guerre, en y faisant venir (ainsi que l'actuel intendant du Canada, M. Raudot, m'a dit que c'était l'intention du roi de France ${ }^{40}$ ) 20,000 hommes, qui préféreront gagner leur vie au bout du fusil plutôt que de labourer la terre, ils pourront sans peine forcer les Anglais à se servir de leur flotte pour se retirer ailleurs, abandonnant à leurs voisins plus forts les fruits de leur travaux.

On ne sait comment un tel mémoire eût été accueilli au commencement de la guerre. En 1708 , il arrivait à point. Le Conseil du commerce l'étudia avec le plus vif intérêt, manifesta le désir de conférer avec son auteur et soumit ses propositions à la reine, avec ce commentaire où se résumait la pensée des

40 Vetch, qui était déjà venu au Canada en 1701 ou en 1702, y revint en août 1705, accompagné du fils du gouverneur du Massachusetts, William Dudley. Chargé d'une mission diplomatique auprès de Vaudreuil, il en profita pour se renseigner de son mieux sur la colonie, tout en pratiquant suffisamment le commerce interlope pour être trouvé coupable de contrebande par l'assemblée législative de sa province. Voir Hammang, The Marquis de Vaudreuil, 129-138. 
conseillers: "Nous prenons la liberté de déclarer que ce serait favoriser grandement le bien-être et la sécurité des sujets de Votre Majesté en Amériqque, en même temps qu'accroître ce commerce si profitable à la Grande-Bretagne, que de chasser les Français de leurs établissements du continent septentrional. » ${ }^{41}$ Le gouvernement anglais s'inquiéta de trouver les moyens de mettre en œuvre la stratégie inspirée par Vetch. ${ }^{42}$

Au printemps de 1709, sa résolution est prise. Dans un message aux gouverneurs d'Amérique la reine annonce qu'elle enverra une expédition au Nouveau Monde pour conquérir les colonies françaises, "conformément aux propositions que nous a soumises notre fidèle et bien-aimé colonel Vetch ${ }^{43}$ Celui-ci doit rallier immédiatement New-York pour distribuer les ordres de la Cour aux administrateurs coloniaux. Dans les instructions qu'elle lui confie, la souveraine réitère son intention de «délivrer » les colons anglais du «voisinage des Français du Canada ». Elle prévoit les mesures propres à assurer l'occupation militaire de Montréal et de Québec. Elle s'engage à distribuer les dépouilles des vaincus - leur sol et leur commerce - à celles des colonies qui auront manifesté le plus d'empressement à collaborer à la conquête. ${ }^{44}$

Accompagné de Francis Nicholson, Vetch débarque à Boston au commencement de mai. Aussitôt, des messagers partent à destination des capitales du Rhode-Island, du Connecticut, du New-York, du New-Jersey et de la Pennsylvanie. Une vague d'enthousiasme guerrier marque la mise en train de la campagne. «Partout, les gouvernements [coloniaux] donnent la cordiale assurance de leur concours. » Ils promettent des contingents qui dépassent largement les prévisions des stratèges impériaux. Vetch et Nicholson rapportent: «Selon toute probabilité, rien ne saurait occasionner l'échec de l'entreprise, si la flotte

41 Le Council of Trade and Plantations à Sunderland, $1^{\text {er }}$ décembre 1708, CSPCS, 25 (1): 164s. Voir ibid., 57, 64.

42 « An explanatory supplement to Capt. Vetch's proposal for an attack upon Quebec and Montreal, together with a scheme for raising the two new battalions required,》 [17 novembre] 1708, ibid., 147-150.

43 Voir la reine Anne à Cornbury, $1^{\text {er }}$ mars 1709, ibid., 232.

44 « H. M. Instructions to Colonel Vetch,» 7 mars 1709 , ibid., 230-232. 
d'Angleterre n'arrive pas trop tard. ${ }^{45}$ Une seule ombre au tableau: les New-Yorkais sont divisés. Sans doute envoient-ils, sous Nicholson, un corps expéditionnaire chargé de marcher sur Montréal, pendant que les opérations principales se déroulent devant Québec; toutefois, la population n'est pas unanime à vouloir la guerre: "Nous avons des gens qui s'y opposent, mais ils sont en minorité. Si l'intérêt mène le monde, il tyrannise le New-York. À Albany, où il se fait du commerce avec les Français du Canada, les trafiquants s'opposent à la participation au conflit, tandis que les ruraux la favorisent. ${ }^{46}$

Ce désagrément fait place à une appréhension autrement grave, à mesure que le temps passe. L'escadre anglaise ne paraît pas. En septembre, Dudley ordonne un jour de jeûne dans le Massachusetts pour hâter la venue de la flotte. ${ }^{47}$ Il ignore encore que, le 8 août, le gouvernement métropolitain a prévenu les colonies de ne pas attendre les effectifs promis; au dernier moment, il a fallu changer leur destination et les utiliser en Europe. La triste nouvelle atteint Boston le 22 octobre. ${ }^{48}$ Tout de suite, une conférence intercoloniale réunit les gouverneurs du Massachusetts, du New-Hampshire, du Connecticut et du Rhode-Island, outre Vetch, Nicholson, des députés et des conseillers. Ce «congrès » expédie à la reine un message de regret. Il demande aussi que l'expédition soit reprise le printemps suivant. Mais, d'ici là, l'Angleterre peut conclure la paix avec la France. Des négociations n'ont-elles pas déjà été entamées ? Que la métropole ne signe pas de traité sans se faire céder le Canada et l'Acadie ! Il est indispensable que l'empire anglais mette au moins la main sur Port-Royal, ce nid de corsaires venus de partout, surtout des Antilles. Si le Canada et l'Acadie allaient rester en la possession de la France, ces deux colonies deviendraient un jour peuplées au point qu'elles se révéleraient extrêmement difficiles à réduire. ${ }^{49}$

45 Nicholson et Vetch à [Sunderland ?], 28 juin 1709, ibid., 399-406.

46 Th. Cockerill à Popple, 2 juillet 1709, ibid., 409.

47 CSPCS, 25 (2) : 30.

48 Ibid., 25 (1): 488.

49 « Address of the Governors of the Massachusetts Bay, New Hampshire, Connecticott and Rhode Island at a Congresse with Col. Nicholson, 
C'est ce que répètent les assemblées législatives du Massachusetts et du New-Hampshire. ${ }^{50}$ Soixante-sept bourgeois et commerçants de Boston mettent leur signature au bas d'une pétition priant la reine de faire en sorte qu'à la paix «la NouvelleÉcosse soit réservée et annexée à vos établissements britanniques ». Ils la supplient, en même temps, de ne pas oublier le Canada. Leur vœu le plus cher serait de voir «l'Amérique, aussi bien que l'Europe, libérée de la tyrannie française $» .51$ Il n'est pas sans importance de comprendre ce que veulent exactement les Bostonnais. Ils ne demandent pas seulement à la métropole d'expulser la France de l'Amérique. Ils désirent que la NouvelleFrance leur soit « réservée et annexée ». En d'autres termes, ils attendent de la mère-patrie qu'elle effectue pour eux la conquête de l'empire rival. Peut-être ne serait-il pas exagéré de voir dans cette ambition le germe des malentendus qui provoqueront, dans le monde anglais, un débat si retentissant à la veille du traité de Paris.

L'année 1710 ne verra pas l'anéantissement du Canada. Elle sera cependant témoin de la chute de l'Acadie. À la fin de 1709, Nicholson était parti pour Londres avec la mission de plaider la cause de la Nouvelle-Angleterre, plus désireuse que jamais d'avoir le champ libre en Amérique. ${ }^{52}$ Ensuite, les colonies avaient attendu avec une impatience croissante les nouvelles de la métropole. À la fin de mai, Vetch rappelait à ses chefs que les provinces américaines n'avaient rien épargné, l'année précédente, pour assurer le succès de l'invasion des territoires ennemis; cette année encore, elles se livraient, dans le même but, à des dépenses considérables. Les retards apportés à la réalisation des projets de conquête avaient pour effet de mettre l'adversaire sur ses gardes. Non seulement les Français se fortifiaient-ils,

Col. Vetch and several Members of the Council and Representatives to the Queen,» 14 octobre 1709 , ibid., 491s.

50 《Resolution of the House of Representatives of the Massachusetts Bay, 》 27 octobre 1709, ibid., 500; "Address of the Governor, Council and Assembly of New Hampshire to the Queen,» 6 décembre 1709, ibid., 25 (2) : 31.

51 Ibid., 25 (1) : 493.

52 Dudley, Vetch et Moody à [Sunderland ?], 25 octobre 1709, ibid., 497; Addington à Popple, 26 octobre 1709, ibid., 499. 
mais ils passaient à l'offensive sur mer: en cinq jours, leurs corsaires venaient de capturer neuf navires au large de Boston. Il restera, prédisait Vetch, "presque impossible d'empêcher de tels malheurs aussi longtemps que les Français posséderont PortRoyal $\gg{ }^{53}$ Les incursions indigènes avaient repris sur la frontière..$^{54}$ En juin, Dudley proclamait un jour de jeûne et de prière «pour que les ordres de Sa Majesté nous parviennent sans délai et soient suivis de succès $\gg .5 \bar{A}$ A la mi-juillet, Nicholson parut à Boston avec six navires. C'était la première division d'une escadre destinée à remettre sur pied l'entreprise manquée en 1709 . À la fin d'août, cependant, on devait recevoir l'avis que, retenus par des vents contraires, les autres vaisseaux ne pourraient pas venir rallier les premiers éléments de la flotte. Les forces dont Nicholson disposait n'eussent rien fait devant Québec. Il les dirigea sur l'Acadie, où il enleva Port-Royal le 13 octobre. ${ }^{56}$

Une colonie française vient de tomber dans l'empire anglais pour n'en plus sortir. De Port-Royal, devenu Annapolis Royal, les vainqueurs préviennent le gouverneur général de la NouvelleFrance que tous les Acadiens, sauf ceux de la capitale et des environs, qui bénéficient d'une capitulation, sont désormais prisonniers des Anglais, à l'absolue discrétion de «S. M. Anne, par la grâce de Dieu reine de Grande-Bretagne, de France et d'Irlande ». ${ }^{57}$ Que signifie ce langage ? Resté sur les lieux à la tête des troupes d'occupation, Vetch explique que les Anglais n'ont pris aucun engagement envers les vaincus, à la réserve de ceux de Port-Royal. Par conséquent, il serait expédient que l'Angleterre envoyât des vaisseaux en vue de procéder à la déportation immédiate de la population, sans quoi «nous ne pouvons jamais compter sur la paisible possession des lieux ».58 Les hommes de 1755 ne raisonneront pas autrement. - Parmi les premiers à féliciter la reine de cette conquête, se présentent à la cour de St.

53 CSPCS, 25 (2) : 101; voir ibid., 25.

54 The Boston News-Letter, 12 et 26 juin 1710 .

55 Ibid., 12 juin 1710.

56 Gerald S. Graham, éd., The Walker Expedition to Quebec, 1711 (The Publications of the Champlain Society XXXII, Toronto, 1953), introduction 10s. Voir Hammang, The Marquis de Vaudreuil, 155s.

57 CSPCS, 25 (2): 230s.

58 Vetch à Dartmouth, 22 janvier 1711, ibid., 343s. 
James des « chevaliers, des marchands et d'autres personnalités éminentes en relations d'affaires avec la province » du Massachusetts. $^{59}$ Tout de suite, s'attache une signification économique à cette nouvelle étape de l'expansion britannique au Nouveau Monde.

\section{L'EXPÉDITION DE WALKER: 1711}

Les commerçants de Londres peuvent se montrer satisfaits, les hommes politiques de la Nouvelle-Angleterre sont loin de s'estimer comblés. Au lendemain de la chute de l'Acadie, le gouverneur, le conseil et l'assemblée législative du Massachusetts réitèrent à la souveraine leur représentation que la réduction du Canada reste «absolument nécessaire » à la tranquillité des provinces septentrionales de l'Amérique anglaise. Pour cette œuvre d'envergure, il faut que la métropole dépêche une flotte et une armée; il faut aussi qu'elle s'assure le concours de toutes ses colonies, y compris la Virginie. ${ }^{60}$ L'agent de la province à la Cour, Jeremiah Dummer, explique de son côté que les motifs qui ont dicté la conquête de l'Acadie sont également valables pour celle du Canada. Quelques-uns, reconnaît-il, sont déjà tentés de juger trop avancée l'économie de l'Amérique britannique; ils appréhendent que « certaines des colonies continentales ne s'adonnent un jour à la fabrication des lainages et n'en deviennent moins avantageuses à la couronne ». Voilà un risque que la métropole ne courra jamais au Canada, pays «extrêmement froid, couvert de neige durant la moitié de l'année et, par conséquent, impropre à l'élevage du mouton ». D'ailleurs, le Canada continue «d'alarmer tout le continent anglais, malgré l'heureuse conquête de Port-Royal... Si bien qu'il ne faut s'attendre à nul repos tant que le Canada, la Carthage d'Amérique, n'aura pas été soumis. Mais une fois qu'on l'aura pris, l'empire britannique d'Amérique connaîtra la liberté et la prospérité; par là, la couronne de la Grande-Bretagne peut un jour être aussi opulente

59 Nouvelle datée du 17 janvier 1711, The Boston News-Letter, 11 juin 1711.

60 Message du 11 novembre 1710, CSPCS, 25 (2) : 254s. 
et aussi puissante dans les Indes Occidentales que le sont les Etats de Hollande dans les Indes Orientales ${ }^{61}$

Sous l'inspiration de Henry St. John, le futur vicomte Bolingbroke, le cabinet se rend soudainement aux désirs impérialistes de la Nouvelle-Angleterre. Il n'y a pas à douter qu'en prenant la décision de conquérir le Canada, St. John n'ait obéi à des soucis de politique partisane; il est incontestable qu'il ait cherché, par une grande victoire militaire, à donner du lustre au parti tory. ${ }^{62}$ Cela dit, il reste que le politicien a aussi exprimé, dans cette conjoncture, des vues d'homme d'État assez semblables, à certains égards, à celles que William Pitt et ses fidèles soutiendront avec éclat un demi-siècle plus tard. Il serait dommage, réfléchitil, qu'à la fin d'une guerre dont elle a porté le plus lourd du fardeau, l'Angleterre fût la seule nation de la Grande Alliance qui n'en retirât pas des avantages particuliers: «Il est grandement temps de faire pour la Grande-Bretagne quelque chose qui cause à l'ennemi un tort aussi grave et aussi essentiel que tous les autres dommages que lui ont déjà faits les opérations qui ont, jusqu'ici, tourné au seul avantage de nos alliés. » La conquête du Canada est certes une opération qui correspond à cette définition. "Supposez les Français chassés du Canada et la reine maîtresse de tout le continent nord-américain. De telles perspectives s'ouvrent alors que celui qui n'en est pas charmé ne peut qu'être dépourvu de tout sens de l'honneur, de la grandeur et de la prospérité de son pays. » Même s'il est légitime que chaque Américain pense à la province qu'il habite, il lui faut aussi considérer dans toute son ampleur «l'ensemble des intérêts britanniques dans cette partie du monde $\gg^{63}$ Voilà déjà les principaux thèmes que reprendront les meilleurs esprits politiques de l'empire britannique dans les années 1750 . Faut-il admirer le précurseur? Observons plutôt qu'il n'y a pas un grand sujet d'étonnement à voir des conditions semblables provoquer de semblables réactions.

Il n'entre pas dans le cadre de cet article d'étudier en détail l'invasion anglaise de 1711. On ne saurait néanmoins s'empêcher

${ }^{61}$ Dummer à Dartmouth, 3 janvier 1711, dans Graham, éd., The Walker Expedition to Quebec, 1711, 267.

$62 \mathrm{Ibid}$., introduction, $12,14$.

63 St. John à Hunter, 6 février 1711, ibid., 276s. 
d'en souligner un aspect: l'énormité (pour l'époque) des moyens confiés à ses chefs, l'amiral Walker et le général John Hill. Walker eut sous ses ordres 14 vaisseaux de guerre, dont deux de 80 canons, trois de 70 et cinq de 60 ; cette escadre pouvait aligner 858 pièces d'artillerie navale; elle était montée par 5,155 matelots. Outre ces unités de combat, l'amiral reçut en Angleterre une cinquantaine de transports, auxquels Dudley en joignit 17 autres pour embarquer les combattants du Massachusetts. ${ }^{64}$ A Hill, revêtu du commandement des troupes de terre, le gouvernement donna sept régiments aguerris, dont cinq tirés des meilleurs éléments de l'armée opérant dans les Flandres sous Marlborough, qui se cabra en vain. ${ }^{5 .}$ Cela, sans compter un corps de fusiliers marins, sans compter les troupes provinciales du Massachusetts, qui excédèrent de dix pour cent les effectifs prévus, sans compter l'armée de 2,300 hommes qui se trouva prête, en septembre, à quitter Albany à destination de Montréal. ${ }^{66}$ La population coloniale pouvait suivre avec satisfaction l'arrivée des vaisseaux et des régiments, les déplacements des chefs, la formation des contingents provinciaux, les mouvements des troupes. ${ }^{67}$ Pour l'expédition, l'assemblée du New-York souscrivait $£ 10,000$; celle du New-Jersey, $£ 5,000$ et même celle de la Pennsylvanie, $22,000 .{ }^{68}$ L'empire lançait vraiment un assaut formidable sur le Canada.

Au début d'avril, Dudley avait proclamé un jour de jeûne en prévision des opérations que, déjà, il anticipait. ${ }^{69}$ À la fin de juillet, il ordonne qu'on en observe un à tous les derniers jeudis du mois tant que durera la campagne..$^{70}$ À l'ouverture de la session de 1711, il annonce aux deux chambres, avec enthousiasme, l'arrivée prochaine d'un gros corps expéditionnaire. ${ }^{71}$ À la mi-juillet, il

64 «By Sir Hovenden Walker Knt. \&c. The Line of Battel,» ibid., 216 ; «The following Lists sent me by the Governour,» ibid., 215.

65 Ibid., introduction, 13.

66 Ibid., 26.

67 The Boston News-Letter, 2 juillet, 23 juillet, 30 juillet, 6 août, 20 août, 10 septembre 1711.

68 Ibid., 23 et 30 juillet 1711.

69 Ibid., 2 avril 1711.

70 The Boston News-Letter, 23 juillet 1711.

71 Ibid., 4 juin 1711. 
convoque de nouveau les législateurs, les priant de seconder les militaires, maintenant que, «par la grâce du Dieu tout-puissant, la flotte et les troupes de Sa Majesté sont arrivées et que les préparatifs sont en train pour l'expédition que nos colonies demandent depuis si longtemps à la reine $\gg^{72}$ La chambre basse remercie la mère-patrie d'avoir eu égard aux «grandes pertes » que les Français et les «Indiens sauvages » ont déjà occasionnées à sa colonie et la félicite de prendre des mesures propres à procurer «l'heureuse tranquillité et le repos durable» à ses sujets d'Amérique en entreprenant d'éliminer leurs ennemis. ${ }^{73}$ Le gouvernement du New-Hampshire exprime les mêmes sentiments; il ajoute: "Ce sera une glorieuse acquisition pour la couronne impériale de la Grande-Bretagne, un bénéfice et un avantage incalculable pour tout l'empire britannique.» ${ }^{74}$

L'opposition métropolitaine s'émeut, elle aussi. Dans le Journal qu'il publiera pour défendre sa conduite, le malheureux Walker reconnaîtra, dès les premières lignes: «L'expédition du Canada a fait autant de bruit à Londres que si le sort de toute la Grande-Bretagne y avait été suspendu. ${ }^{75}$ Dans la capitale anglaise, un périodique publie un long article sur la NouvelleFrance: "Parce que l'expédition actuellement en route vers Québec est une entreprise d'une telle importance pour la GrandeBretagne et qu'elle fait le sujet des conversations, nous pensons qu'on nous approuvera de présenter au lecteur un aperçu du Canada... et surtout de la ville de Québec. » A l'exemple, y lit-on, des habitants de l'Ancienne France, ceux de la Nouvelle se sont toujours évertués à «usurper les possessions des Anglais ». Ils ont trouvé le moyen de dépouiller ces derniers de la baie d'Hudson cependant que, par les artifices de leur diplomatie, ils ont soulevé les indigènes contre la Nouvelle-Angleterre. «Pendant la dernière guerre [de la ligue d'Augsbourg], on a mis sur pied une couple d'entreprises contre Québec. Mais la

72 Ibid., 9 juillet 1711.

73 « Resolution by the House of Representatives of the Massachusetts Bay,» Graham, éd., The Walker Expedition to Quebec, 1711, 300.

74 «Address of the Governor, Council and Assembly of New Hampshire to the Queen,》 22 juillet 1711 , ibid., 309 .

75 Ibid., 57. 
conquête de la Nouvelle-France, aussi bien que la réduction de l'Ancienne à ses justes dimensions, était, semble-t-il, réservée au règne glorieux de la présente reine, dont les troupes s'emparèrent, l'année passée, de Port-Royal, en Acadie. Je pourrais ici développer les heureuses conséquences de l'expédition en cours pour l'expansion de notre commerce et la reprise de certaines de ses manifestations, qui semblaient irrévocablement perdues. $\gg^{76}$ Peu auparavant, la souveraine avait justement donné sa sanction à une législation intitulée: «Loi en vue d'encourager le commerce avec l'Amérique.»77

Pendant que l'Angleterre et ses colonies supputent les profits de leur prochaine victoire, quel sort se disposent-elles à faire aux Canadiens ? Avant de prendre la mer, le général Hill a fait imprimer à Boston une proclamation qu'il se propose de distribuer dans le pays ennemi. La Grande-Bretagne, y déclaret-il, détient « des droits et des titres justes sur toute l'Amérique Septentrionale, par la premiere decouverte qui en a ete faite et par la possession». De plus, elle est autorisée à des compensations, à la suite «des horribles barbaries et des cruautez inouies, excitees et commises par les François avec les Indiens » contre «ses Sujets opprimés d'une maniere si abominable». Tout bien considéré, les habitants de la Nouvelle-France sont «aussi bien Sujets de la Couronne de la Grande Bretagne, que s'ils y etoient nez et etablis, ou en Irlande, ou en d'autres endroits des Colonies de Sa Majesté, qui sont immediatement sous sa Protection». Ceux des Canadiens qui se soumettront sans résistance «seront favorablement receus et traitez, et continuez eux et leurs heritiers, en une douce et paisible possession de leurs terres, maisons et autres biens ... et jouiront de la liberté, des privileges et exemtions en commun avec le reste des Sujets naturels de Sa Majesté, avec le libre exercice de leur Religion». Ceux qui préféreront passer en France en recevront les moyens. Si le clergé exhorte les fidèles à se rendre de bonne grâce aux envahisseurs, ceux-ci auront pour ses membres «toute sorte de

76 Article daté de Londres, 6 octobre 1711, reproduit dans la Boston News-Letter, 4 août 1712.

77 Dépếche datée de Londres, 12 juin 1711, dans la Boston News-Letter, 3 septembre 1711 . 
considération » et leur permettront de se transporter en France «avec les effets qui paroitront leur appartenir». En revanche, les Canadiens coupables de s'opposer aux Anglais seront traités en ennemis et leurs biens confisqués au profit de la reine «pour etre distribuez a ceux qui donneront quelque assistance, afin que ces pays soient sous la Domination de sa Majesté de la Grande Bretagne». Les résistants seront impitoyablement «domptes par la force des armes $\gg{ }^{78}$

Ces beaux projets allèrent se briser, dans la nuit du 2 au 3 septembre 1711, avec huit des transports de l'escadre, sur les masses de granit de la Côte nord, vers l'île aux Oeufs ou la pointe aux Anglais. Quatre semaines plus tard, la News-Letter de Boston publie, en texte très fin, une brève nouvelle révélant la perte des navires. Au bout d'un autre mois, elle rapporte qu'un jour de pénitence vient d'être « rigoureusement observé ... à la suite du malheur qui s'est abattu sur la flotte au cours de l'expédition ${ }^{79}$ Sur les entrefaites, une dépêche de NewYork mande que Nicholson, déjà en marche sur Montréal, a eu avis du « désastre » survenu dans le Saint-Laurent. Il lui faudra rebrousser chemin sans avoir rien fait. ${ }^{80}$ Dans un discours prononcé devant les deux chambres de sa province, puis dans une proclamation, Dudley exhale d'amers regrets. Maintenant, s'écrie-t-il, «nous savons tous ... à quoi nous devons nous attendre de la part de nos ennemis $\gg .81$ Les Acadiens et les Indiens, enchaîne Vetch, avaient esquissé des gestes de «soumission »; c'est qu'ils étaient persuadés de l'effondrement prochain du Canada: «Mais, dès qu'ils auront appris notre désastre et reçu de nouveaux ordres du Canada, je ne doute pas que nous n'ayons sur les bras tous les éléments français et indigènes que l'on pourra

78 « De Par Son Excellence, Monsieur Jean Hill, General et Commandant en chef les Troupes de sa Majesté Bretanique en Amerique 》Archives du Séminaire de Québec, Documents Faribault, no 132. - L'exemplaire conservé au Séminaire de Québec porte, en marge, ces mots de la main de Walker: «Monsr: Jean de La Fotte a le Pouvoir de promettre de ma part Ces Condition Ici exprime Seront egalement pour Les Habitants de L'Acadie. fait 10/21 Aoust 1711 Hovenden Walker Amiral.» — L'auteur désire remercier le Séminaire de Québec de lui avoir ouvert ses archives. 79 Numéros du 17 septembre, 24 septembre et 15 octobre 1711.

80 The Boston News-Letter, 1er et 8 octobre 1711.

81 Ibid., 29 octobre et 19 novembre 1711 . 
mobiliser contre notre garnison [Port-Royal], au début du printemps, sinon plus tôt. ${ }^{82}$

A vrai dire, les hostilités n'avaient jamais entièrement cessé en Acadie. Des délégations indigènes étaient allées parler de paix à Annapolis, au printemps, ${ }^{83}$ mais, outre que plusieurs incursions sanglantes avaient secoué des agglomérations de la Nouvelle-Angleterre, ${ }^{84}$ un fort détachement était venu, à la mijuin, opérer jusque dans le voisinage de l'ancien Port-Royal, où il avait surpris un groupe de soixante Anglais: plusieurs avaient été massacrés sur place et trente-quatre capturés pour être expédiés à Québec. ${ }^{85}$ Après la déconfiture de Walker, on craignait que les Canadiens ne s'enhardissent au point d'attaquer le New-York. ${ }^{86}$ A la fin d'octobre, des colons se faisaient tuer à dix-huit milles d'Albany. ${ }^{87}$ Ce fut cependant la NouvelleAngleterre qui eut le plus à souffrir. Comme il avait été aisé de le prévoir, le printemps de 1712 fut affreux. Un bulletin de Piscataqua mande, vers la mi-mai: "Il ne se passe presque pas de jour que l'ennemi ne nous inflige quelque dégât. ${ }^{88}$ L'été n'apporte guère de répit. ${ }^{89}$ L'heure n'était pas encore venue pour les Anglais de goûter un «repos durable » en Amérique.

Le Massachusetts ne voulait toutefois pas abandonner tout espoir de conquête et de suprématie indiscutée. A la fin d'octobre 1711, son gouvernement persistait à croire que la reine pourrait bien, «dans sa sagesse princière », envoyer une autre escadre et un nouveau corps expéditionnaire en $1712 .{ }^{90}$ Le NewHampshire s'entêtait, lui aussi, à partager cette espérance. ${ }^{91}$ Le

82 Vetch à Dartmouth, 16 novembre 1711, Graham, éd., The Walker Expedition to Quebec, 1711, 383.

83 The Boston News-Letter, 26 mars 1711.

84 Ibid., 9 avril, 7 mai et 14 mai 1711 .

85 Ibid., 25 juin 1711.

86 The Boston News-Letter, 15 octobre 1711.

87 Ibid., 5 novembre 1711.

88 Ibid., 12 mai 1712 . Voir ibid., 21 avril, 28 avril, 5 mai, 19 mai, 2 juin, 9 juin 1712 .

89 Ibid., 14 juillet, 21 juillet, 28 juillet, 8 septembre, 22 septembre, 29 septembre 1712 , etc.

90 «Address of the Governor, Council and Assembly of the Massachusetts Bay to the Queen,» 17 octobre 1711, Graham, éd., The Walker Expedition to Quebec, 1711,370 .

91 « Address of the Governor, Council and Representatives of New Hampshire to the Queen,» 30 octobre 1711, ibid., 371s. 
conseil et l'assemblée du New-York suppliaient la souveraine de renouveler la tentative. ${ }^{92}$ Le gouverneur du Connecticut évoquait les massacres que les indigènes ne manqueraient pas de perpétrer dans sa province; il lançait un appel à la «compassion» du cabinet métropolitain, tout en faisant miroiter à ses yeux « le grand avantage que la réduction des établissements français de l'Amérique du Nord doit procurer à la couronne $\gg .^{93}$ Walker lui-même songeait à se reprendre. Il écrivait après le naufrage: « Je crois avoir découvert la force et la faiblesse réelles des Français de l'Amérique du Nord et je serai en état, quand il plaira à Dieu de me ramener sain et sauf en Grande-Bretagne, d'élaborer, pour nous soumettre ces gens-là, de meilleurs plans que ceux que l'on a faits jusqu'à ce jour. $\gg^{94}$

\section{CONCLUSION}

Walker ne reviendra pas. La dernière nouvelle que la NewsLetter de Boston donne de son escadre mande que celle-ci est rentrée à Portsmouth, où, quelques jours après avoir accosté, le vaisseau amiral, l'Edgar, a fait explosion avec presque tout son équipage à bord. Dans le même numéro, on lit qu'un congrès doit s'ouvrir à Utrecht le 12 janvier $1712 .{ }^{95}$ Les pourparlers de paix accapareront désormais l'attention.

A la rentrée des chambres anglaises, en décembre 1711, le message royal proclame la volonté de procurer « une bonne paix » à la nation. "Je ferai en sorte, déclare la souveraine, qu'après une guerre qui a coûté tant de sang et d'argent, le traité de paix assure l'amélioration et l'expansion de vos intérêts commerciaux. $\gg^{96}$ Dans un autre message à la chambre des Lords, à la fin de janvier 1712 , la reine annonce l'arrivée de ses plénipotentiaires à Utrecht. Ceux-ci, souligne-t-elle, ont entamé des conversations destinées à faire obtenir satisfaction à tous les alliés, "particulièrement en ce qui concerne l'Espagne et les

92 Hunter à Dartmouth, 12 novembre 1711, ibid., 375.

93 Saltonstall au Lord High Treasurer, 12 novembre 1711 , ibid., 376.

94 Walker à St. John, 12 septembre 1711, ibid., 358 .

95 Numéro du 25 février 1712.

96 The Boston News-Letter, 14 avril 1712. 
Indes Occidentales ${ }^{97}$ Les exigences de la Grande-Bretagne sont connues en Amérique dans les derniers jours de mai. L'Angleterre veut que la France lui cède les îles de Saint-Christophe et de Terre-Neuve. Elle réclame «l'Acadie, avec la ville de PortRoyal, autrement appelée Annapolis Royal, ainsi que le territoire qui en dépend ». Elle revendique la baie d'Hudson. Elle entend que les Canadiens s'abstiennent dorénavant «d'entraver le trafic réciproque entre les sujets de la Grande-Bretagne et les indigènes de l'Amérique comme aussi d'inquiéter les cinq Nations ou Cantons indiens et autres tribus qui relèvent de son autorité ».98 Un autre discours de la reine Anne aux Lords et aux Communes, en juin 1712, fait ressortir les visées du cabinet anglais au Nouveau Monde: «Nous avons un intérêt si profond au commerce de l'Amérique du Nord que j'ai fait les plus vifs efforts pour tourner cet article à notre plus grand avantage. La France consent à nous rendre toute la baie et le détroit d'Hudson, à nous remettre l'île de Terre-Neuve avec Plaisance et à effectuer la cession absolue d'Annapolis avec le reste de la Nouvelle-Ecosse ou Acadie. $\gg^{99}$

Venant à la suite de celles que nous avons déjà retenues, ces diverses déclarations nous indiquent jusqu'à quel point expansion coloniale et commerce sont liés dans l'esprit des hommes d'Etat britanniques. Ceux-ci donnent l'impression très nette qu'à leurs yeux, mettre la métropole en état d'élargir la base de son trafic colonial, c'est tout gagner. En cela, semble-t-il, ils tombent d'accord avec une fraction influente de l'opinion. Commentant les buts de guerre de la nation à la lumière de la conférence de la paix, le lord-maire et les échevins de Londres s'adressent ainsi à la reine: "Nous, de la Cité, ne connaîtrions ni nos intérêts ni notre devoir si nous n'exprimions pas toute notre reconnaissance à la vue des bénéfices inestimables que nous et notre postérité pouvons espérer de recueillir du soin attentif que Votre Majesté a pris du commerce britannique en lui pro-

97 Ibid., 5 mai 1712.

98 « The Specifick Demands of Her Majesty the Queen of Great Britain, for what relates to France,» bulletin daté d'Utrecht, 8 mars, The Boston News-Letter, 19 mai 1712.

99 Ibid., 6 octobre 1712. 
curant la sûreté là où il avait été troublé, en le rétablissant là où il avait été perdu et en l'étendant à des parties du monde qu'il n'avait pas encore atteintes. ${ }^{1}$

Pourtant, Vetch, Dudley, Dummer et les impérialistes éclairés qui partageaient leurs vues avaient situé la conquête du Canada dans des perspectives qui dépassaient largement celles d'une simple expansion économique. Sans doute avaient-ils souligné les profits qui ne pourraient manquer d'en découler pour le commerce anglais. En quoi ils avaient eu raison. Mais ils n'avaient pas eu le tort de se laisser hypnotiser par des considérations de marchands au point de ne pas voir le reste. Et le reste, c'était l'essentiel. Pour eux, il ne s'agissait pas uniquement, pas même surtout d'entrer en Acadie et au Canada pour s'en approprier les ressources et en ouvrir les marchés aux commerçants d'Angleterre. Il s'agissait d'abord de supprimer l'Acadie et le Canada, d'éliminer - ils l'ont assez répété - ce gênant voisinage afin de faire sauter les obstacles qu'il comportait pour la croissance normale de l'Amérique anglaise. Ils ne disaient pas: il faut exploiter le Canada. Ils clamaient: «Delenda est Carthago!»

Le 12 août 1712, l'Angleterre conclut avec la France une suspension d'armes qui produira l'effet d'entraîner à la paix les autres puissances de la Grande Alliance. En novembre, les gouverneurs des colonies américaines font connaître officiellement l'armistice aux populations. ${ }^{2}$ C'est une "heureuse nouvelle», commente Dudley. ${ }^{3}$ Voilà, écrit le gouverneur du Maryland, «la fin d'une guerre qui nous a atteints si profondément que nous en sortons, pour la plupart, misérablement appauvris et, pour beaucoup, tout à fait ruinés ».4 Signé le 11 avril 1713 , le traité d'Utrecht inspire surtout un sentiment de soulagement aux Américains britanniques. Les Virginiens, qui avaient éprouvé

1 « The Humble Address of the Lord Mayor, Aldermen and Commons of the City of London, in Common-Council assembled," 14 juin 1712, The Boston News-Letter, 6 avril 1713.

2 Ibid., 3 novembre 1712. 27: 103

3 Lettre au Council of Trade and Plantations, 2 décembre 1712, CSPCS, 93.

${ }^{4}$ Lettre au Council of Trade and Plantations, 20 novembre 1712, ibid., 
un mal infini à écouler leur tabac durant le conflit, accueillent la paix avec l'espoir qu'elle les mettra à même de réparer les pertes qu'une "guerre aussi longue et aussi onéreuse » a occasionnées à leur commerce. ${ }^{5}$ A Boston, la proclamation du traité donne lieu à des cérémonies officielles et à « la joie universelle des bons sujets de Sa Majesté ». ${ }^{6}$ Les assemblées législatives du Massachusetts et du Connecticut remercient la métropole de l'acquisition de Terre-Neuve et de la Nouvelle-Écosse. ${ }^{7}$ Au Canada, d'autre part, Vaudreuil parle à la Cour de « la veritable joye que les peuples de ce pays» ont ressentie aux «bonnes nouvelles» de la suspension d'armes, puis du traité d'Utrecht. ${ }^{8}$

L'Amérique britannique avait cru pouvoir détruire l'Amérique française à la faveur de la guerre. La paix lui donnait la baie d'Hudson (dont profitera la Hudson's Bay Company), Terre-Neuve, l'Acadie et, au moyen du protectorat que la France reconnaissait à l'Angleterre sur les Iroquois, le droit de coloniser la région des Grands Lacs. Sauf en Acadie, dont il s'emparait pour délivrer ses établissements septentrionaux de la menace de Port-Royal, l'empire britannique ne semblait s'agrandir que pour mettre la main sur quelque trafic: celui du poisson à l'est, celui des fourrures au nord et dans le Centre-Ouest. Il est sûr que les dispositions du traité d'Utrecht ${ }^{9}$ ont porté un coup extrêmement sensible à la Nouvelle-France. Exécuté à la lettre, le traité eût rompu la charnière des Lacs articulant la Louisiane au Canada. La Nouvelle-France sautait. Normalement, Utrecht aurait dû rétrécir les horizons du Canada jusqu'à le faire déchoir au rang de pays agricole, frappé de rachitisme au milieu de ses vigoureux concurrents américains, et donc irrémédiablement battu d'avance. Mais, seule, une société défaite saurait se résigner à un tel sort. Et le Canada n'était pas défait. Malgré

5 « Address of the Lt. Governor and Council of Virginia to the Queen,» 12 août 1713 , ibid., 221 .

6 Dudley à Popple, 25 août 1713, ibid., 231; The Boston News-Letter, 31 août 1713 .

7 Dudley au Council of Trade and Plantations, 24 août 1713, CSPCS, 27: 225; Saltonstall à [Bolingbroke ?], 2 novembre 1713, ibid., 249.

8 Lettre du 14 novembre 1713, RAPQ (1947-48), 232.

9 Voir les articles du traité d'Utrecht relatifs à l'Amérique dans Histoire du Canada par les textes (Montréal, 1952), 56-59. 
les représentations réitérées de ses colonies américaines, le gouvernement anglais l'avait laissé vivre, satisfait des plantureux avantages commerciaux qu'il avait arrachés aux empires français et espagnol.

Cependant, même en Angleterre, tout le monde n'était pas si content. Fort au courant des mouvements de l'opinion britannique, Vaudreuil donnait au Régent un avertissement qu'il faut retenir :

On ne peut pas douter un instant qu'à la première rupture entre la France \& l'Angleterre les Anglais ne fassent tous leurs efforts pour s'emparer de tout le Canada, \& par conséquent de toute L'Amérique Septentrionale, d'où il pourrait s'ensuivre la perte du Mexique dont ils chasseraient en peu d'années les Espagnols sans aucune résistance.

Ils ont assez fait voir leurs intentions par l'armement qu'ils ont fait en 1711 \& même depuis la paix, par le vingt deuxième article des instructions données par la ville de Londres à ses députés qui porte en termes exprès qu'ils demanderont compte aux ministres du gouvernement précèdent, pourquoi ils ont laissé à la France le Canada \& L'Ile du Cap Breton, à présent appelée L'Ile Royale . . .

On ne peut exprimer à quel point la puissance de l'Angleterre augmenterait si elle s'emparait du reste de l'Amérique Septentrionale, \& combien cette puissance deviendrait redoutable en Europe.

Cela doit assez faire connaître la nécessité qu'il y a de profiter de la paix pour fortifier le Canada... ${ }^{10}$

Vaudreuil voit terriblement juste. En 1713, la fraction de l'opinion anglaise qu'inspire un mercantilisme déjà légèrement arriéré impose à la nation une paix où le commerce trouve son compte, mais qui ne correspond pas suffisamment aux aspirations vitales de l'Amérique britannique. Cinquante ans plus tard, on le sait, la même question se posera. Elle sera longuement débattue. ${ }^{11}$ Cette fois, l'idée impérialiste l'emportera sur

10 «Mémoire de M. de Vaudreuil au duc d'Orléans,» février 1716, RAPQ (1947-48), 292.

11 Voir les chapitres X et XI de La Guerre de la Conquête, 401-454. 
la pensée mercantiliste. L'expérience d'un demi-siècle aura enseigné qu'il est impossible, sans éliminer le Canada, de démembrer la Nouvelle-France et de la réduire à l'impuissance, comme l'exige le développement normal de l'aile américaine de la nation anglaise. En 1708, Vetch avait mesuré le risque qu'il y avait à laisser survivre le Canada. Combien ce risque était réel, rien ne le montre mieux que la belle formule mise en avant par Vaudreuil: "Profiter de la paix pour fortifier le Canada.». En somme, le traité d'Utrecht, qui marque le début de la prépondérance de l'Angleterre, est plein de menaces pour la NouvelleFrance. Il donne toutefois à la métropole française la chance - que Vetch, Dudley, Dummer et leur école ne lui accorderaient pas - de rétablir ses positions au Nouveau Monde. Le danger le plus grave qui pèse sur le Canada demeure la farouche volonté de conquête que la guerre de la Succession d'Espagne a mise en lumière chez ses voisins britanniques. Cette volonté ne désarmera jamais.

Université de Montréal.

GUY FrÉGAULT

EDITIONs Fides. - La guerre de la conquête par Guy Frégault. Ouvrage qui constitue une étude magistrale des dernières années de la Nouvelle-France. Volume relié, 520 pages. Prix: $\$ 5.00$. 